\title{
Front Matter: Volume 9783
}

, "Front Matter: Volume 9783," Proc. SPIE 9783, Medical Imaging 2016:

Physics of Medical Imaging, 978301 (10 June 2016); doi: 10.1117/12.2230171

SPIE. Event: SPIE Medical Imaging, 2016, San Diego, California, United States 


\title{
PROGRESS IN BIOMEDICAL OPTICS AND IMAGING
}

\section{Medical Imaging 2016}

\section{Physics of Medical Imaging}

\author{
Despina Kontos \\ Thomas G. Flohr \\ Joseph Y. Lo \\ Editors
}

28 February - 2 March 2016

San Diego, California, United States

Sponsored by

SPIE

Cosponsored by

Modus Medical Devices Inc. (Canada)

Bruker (United States)

Poco Graphite (United States)

imXPAD (France)

Carestream Health, Inc.

GE Healthcare

Cooperating Organizations

AAPM-American Association of Physicists in Medicine (United States)

APS-American Physiological Society (United States)

IFCARS - International Foundation for Computer Assisted Radiology and Surgery (Germany)

Medical Image Perception Society (United States)

Radiological Society of North America (United States)

Society for Imaging Informatics in Medicine (United States)

World Molecular Imaging Society

The DICOM Standards Committee

Volume 9783

Part One of Three Parts

Proceedings of SPIE, 1605-7422, V. 9783

SPIE is an international society advancing an interdisciplinary approach to the science and application of light.

Medical Imaging 2016: Physics of Medical Imaging, edited by Despina Kontos, Thomas G. Flohr, Joseph Y. Lo,

Proc. of SPIE Vol. 9783, 978301 - (c) 2016 SPIE · CCC code: 1605-7422/16/\$18 · doi: 10.1117/12.2230171 
The papers in this volume were part of the technical conference cited on the cover and title page. Papers were selected and subject to review by the editors and conference program committee. Some conference presentations may not be available for publication. Additional papers and presentation recordings may be available online in the SPIE Digital Library at SPIEDigitallibrary.org.

The papers reflect the work and thoughts of the authors and are published herein as submitted. The publisher is not responsible for the validity of the information or for any outcomes resulting from reliance thereon.

Please use the following format to cite material from this book:

Author(s), "Title of Paper," in Medical Imaging 2016: Physics of Medical Imaging, edited by Despina Kontos, Thomas G. Flohr, Joseph Y. Lo, Proceedings of SPIE Vol. 9783 (SPIE, Bellingham, WA, 2016) Article CID Number.

ISSN: 1605-7422

ISSN: 2410-9045 (electronic)

ISBN: 9781510600188

Published by

SPIE

P.O. Box 10, Bellingham, Washington 98227-0010 USA

Telephone +1 3606763290 (Pacific Time) · Fax +1 3606471445

SPIE.org

Copyright (C) 2016, Society of Photo-Optical Instrumentation Engineers.

Copying of material in this book for internal or personal use, or for the internal or personal use of specific clients, beyond the fair use provisions granted by the U.S. Copyright Law is authorized by SPIE subject to payment of copying fees. The Transactional Reporting Service base fee for this volume is $\$ 18.00$ per article (or portion thereof), which should be paid directly to the Copyright Clearance Center (CCC), 222 Rosewood Drive, Danvers, MA 01923. Payment may also be made electronically through CCC Online at copyright.com. Other copying for republication, resale, advertising or promotion, or any form of systematic or multiple reproduction of any material in this book is prohibited except with permission in writing from the publisher. The CCC fee code is $1605-7422 / 16 / \$ 18.00$.

Printed in the United States of America.

Publication of record for individual papers is online in the SPIE Digital Library.

\section{SPIE. DIGITAL}

Paper Numbering: Proceedings of SPIE follow an e-First publication model, with papers published first online and then in print. Papers are published as they are submitted and meet publication criteria. A unique citation identifier (CID) number is assigned to each article at the time of the first publication. Utilization of CIDs allows articles to be fully citable as soon as they are published online, and connects the same identifier to all online, print, and electronic versions of the publication. SPIE uses a six-digit CID article numbering system in which:

- The first four digits correspond to the SPIE volume number.

- The last two digits indicate publication order within the volume using a Base 36 numbering system employing both numerals and letters. These two-number sets start with 00, 01, 02, 03, 04, $05,06,07,08,09,0 A, 0 B \ldots$ OZ, followed by 10-1Z, 20-2Z, etc.

The CID Number appears on each page of the manuscript. The complete citation is used on the first page, and an abbreviated version on subsequent pages. 


\title{
Contents
}

\section{Part One}

\author{
xvii Authors \\ xxv Conference Committee \\ xxix 2016 Medical Imaging Award Recipients
}

SESSION 1 TOMOSYNTHESIS AND DIGITAL SUBTRACTION ANGIOGRAPHY

978302 Feasibility of reduced-dose 3D/4D-DSA using a weighted edge preserving filter [9783-5]

978303 Quantification of resolution in multiplanar reconstructions for digital breast tomosynthesis [9783-1]

978304 A new generation of stationary digital breast tomosynthesis system with wider angular span and faster scanning time [9783-3]

978305 Stationary digital chest tomosynthesis for coronary artery calcium scoring [9783-2]

978306 Detection of microcalcification clusters by 2D-mammography and narrow and wide angle digital breast tomosynthesis [9783-4]

\section{SESSION 2 BREAST IMAGING}

$978307 \quad$ Estimating breast thickness for dual-energy subtraction in contrast-enhanced digital mammography using calibration phantoms [9783-11]

978308 Generation of 3D synthetic breast tissue [9783-6]

978309 A new, open-source, multi-modality digital breast phantom [9783-10]

9783 OA Rayleigh imaging in spectral mammography (Second Place, Physics of Medical Imaging Student Paper Award) [9783-7]

9783 OB Reproducing 2D breast mammography images with 3D printed phantoms [9783-8]

9783 OC Breast ultrasound tomography with two parallel transducer arrays [9783-9]

\section{SESSION 3 KEYNOTE AND DUAL AND MULTI ENERGY CT}

9783 OD Limited-angle multi-energy CT using joint clustering prior and sparsity regularization [9783-14] 
9783 OE Dictionary-based image denoising for dual energy computed tomography [9783-13]

SESSION 4 CONE BEAM CT I: NEW TECHNOLOGIES, CORRECTIONS

9783 OG Twin robotic x-ray system for 2D radiographic and 3D cone-beam CT imaging [9783-15]

$9783 \mathrm{OH} \quad$ Five-dimensional motion compensation for respiratory and cardiac motion with conebeam CT of the thorax region [9783-18]

9783 Ol Shifted detector super short scan reconstruction for the rotate-plus-shift trajectories and its application to C-arm CT systems [9783-20]

9783 0J Striped ratio grids for scatter estimation [9783-17]

9783 OK Image-based motion compensation for high-resolution extremities cone-beam CT [9783-16]

$9783 \mathrm{OL} \quad$ Over-exposure correction in knee cone-beam CT imaging with automatic exposure control using a partial low dose scan [9783-19]

\section{SESSION 5 PHASE CONTRAST IMAGING}

$97830 \mathrm{M} \quad \mathrm{X}$-ray differential phase contrast imaging using a grating interferometer and a single photon counting detector [9783-22]

$9783 \mathrm{ON} \quad$ Potential use of microbubbles (MBs) as contrast material in x-ray dark field (DF) imaging: How does the DF signal change with the characteristic parameters of the MBs? [9783-23]

9783 OP High-energy x-ray grating-based phase-contrast radiography of human anatomy [9783-25]

$97830 Q \quad$ Single-shot $x$-ray phase contrast imaging with an algorithmic approach using spectral detection [9783-21]

\section{SESSION 6 CONE BEAM CT II: SYSTEM OPTIMIZATION, IMAGE RECONSTRUCTION}

9783 OR Nonlinear statistical reconstruction for flat-panel cone-beam CT with blur and correlated noise models [9783-29]

9783 OS Automatic intrinsic cardiac and respiratory gating from cone-beam CT scans of the thorax region [9783-30]

9783 ОT Design and characterization of a dedicated cone-beam CT scanner for detection of acute intracranial hemorrhage [9783-26]

9783 OU C-arm cone beam CT perfusion imaging using the SMART-RECON algorithm to improve temporal sampling density and temporal resolution [9783-28] 
9783 OV Mask free intravenous 3D digital subtraction angiography (IV 3D-DSA) from a single C-arm acquisition [9783-27]

9783 OW Reduction of beam hardening artifacts in cone-beam CT imaging via SMART-RECON algorithm [9783-31]

\section{SESSION 7 CT I: TECHNOLOGY, SYSTEM CHARACTERIZATION, APPLICATIONS}

9783 OX Fluence-field modulated x-ray CT using multiple aperture devices [9783-33]

9783 OY Development of a realistic, dynamic digital brain phantom for CT perfusion validation [9783-32]

$97830 Z$ Experimental characterization of extra-focal radiation in CT scanners [9783-36]

$978310 \quad$ Noise characteristics of CT perfusion imaging: how does noise propagate from source images to final perfusion maps? [9783-35]

978311 Accuracy of patient specific organ-dose estimates obtained using an automated image segmentation algorithm [9783-34]

978312 Dual-source multi-energy CT with triple or quadruple x-ray beams [9783-37]

978313 Optimized projection binning for improved helical amplitude- and phase-based 4DCT reconstruction in the presence of breathing irregularity [9783-38]

\section{SESSION 8 DETECTORS}

978314 DQE simulation of a-Se x-ray detectors using ARTEMIS [9783-42]

978315 Improving detector spatial resolution using pixelated scintillators with a barrier rib structure [9783-39]

978316 High dynamic range CMOS-based mammography detector for FFDM and DBT [9783-43]

978317 Solid-state flat panel imager with avalanche amorphous selenium (Second Place, Physics of Medical Imaging Student Paper Award) [9783-40]

978318 A novel x-ray detector design with higher DQE and reduced aliasing: Theoretical analysis of $x$-ray reabsoprtion in detector converter material [9783-41]

\section{SESSION 9 CT II: IMAGE RECONSTRUCTION, ARTIFACT REDUCTION}

978319 A generalized Fourier penalty in prior-image-based reconstruction for cross-platform imaging [9783-45]

9783 1A Reduction of motion artifacts in cardiac CT based on partial angle reconstructions from short scan data [9783-49] 
9783 1B An open library of CT patient projection data [9783-44]

9783 1C Limits to dose reduction from iterative reconstruction and the effect of through-slice blurring [9783-46]

9783 1D Reduction of truncation artifacts in CT images via a discriminative dictionary representation method [9783-48]

9783 1E Compensation of skull motion and breathing motion in CT using data-based and imagebased metrics, respectively [9783-47]

\section{SESSION 10 PHOTON COUNTING CT I: INSTRUMENTATION}

$9783 \mathrm{IF} \quad$ Estimation of signal and noise for a whole-body photon counting research CT system [9783-50]

9783 1G Material decomposition and virtual non-contrast imaging in photon counting computed tomography: an animal study [9783-51]

$97831 \mathrm{H} \quad$ On the analogy between pulse-pile-up in energy-sensitive, photon-counting detectors and level-crossing of shot noise [9783-52]

$978311 \quad$ A high-resolution imaging technique using a whole-body, research photon counting detector CT system [9783-54]

$97831 \mathrm{~J} \quad$ Lossless compression of projection data from photon counting detectors [9783-53]

\section{SESSION 11 PET AND MR}

$97831 \mathrm{~K}$ Initial experience in primal-dual optimization reconstruction from sparse-PET patient data [9783-58]

$9783 \mathrm{lL} \quad$ Short term reproducibility of a high contrast 3-D isotropic optic nerve imaging sequence in healthy controls [9783-55]

$97831 \mathrm{M} \quad$ Crystal timing offset calibration method for time of flight PET scanners [9783-56]

$97831 \mathrm{~N} \quad$ Solving outside-axial-field-of-view scatter correction problem in PET via digital experimentation [9783-57]

\section{SESSION 12 PHOTON COUNTING CT II: SPECTRAL IMAGING}

978310 Improving material decomposition by spectral optimization of photon counting computed tomography [9783-62]

9783 IP Optimal selection of thresholds for photon counting CT [9783-63]

$97831 Q \quad$ "Conventional” CT images from spectral measurements [9783-61] 
9783 IR Spatio-energetic cross-talks in photon counting detectors: detector model and correlated Poisson data generator [9783-59]

9783 is Comparison of quantitative k-edge empirical estimators using an energy-resolved photoncounting detector [9783-60]

\section{SESSION 13 NEW SYSTEMS AND TECHNOLOGIES}

$97831 \mathrm{U}$ Multi-gamma-source CT imaging system: a feasibility study with the Poisson noise [9783-65]

9783 IV High-spatial-resolution nanoparticle x-ray fluorescence tomography [9783-67]

9783 IW Investigation of noise and contrast sensitivity of an electron multiplying charge-coupled device (EMCCD) based cone beam micro-CT system [9783-66]

9783 1X 800-MeV magnetic-focused flash proton radiography for high-contrast imaging of lowdensity biologically-relevant targets using an inverse-scatter collimator [9783-69]

$97831 \mathrm{Y}$ Method for dose-reduced 3D catheter tracking on a scanning-beam digital x-ray system using dynamic electronic collimation [9783-64]

\section{SESSION 14 SCATTER AND DIFFRACTION IMAGING}

978312 Method to study sample object size limit of small-angle x-ray scattering computed tomography [9783-70]

978321 Coded aperture coherent scatter imaging for breast cancer detection: a Monte Carlo evaluation [9783-74]

978323 Coded aperture x-ray diffraction imaging with transmission computed tomography sideinformation [9783-73]

\section{SESSION 15 TASK DRIVEN IMAGING, OBSERVERS, DETECTABILITY, PHANTOM STUDIES}

978324 Task-driven tube current modulation and regularization design in computed tomography with penalized-likelihood reconstruction [9783-75]

978325 Comparison of model and human observer performance in FFDM, DBT, and synthetic mammography [9783-80]

978326 Predicting detection performance with model observers: Fourier domain or spatial domain? [9783-79]

978327 Investigation of optimal parameters for penalized maximum-likelihood reconstruction applied to iodinated contrast-enhanced breast CT [9783-78] 
978328 Design, fabrication, and implementation of voxel-based 3D printed textured phantoms for task-based image quality assessment in CT (Second Place, Robert F. Wagner AllConference Best Student Paper Award) [9783-76]

978329 Detectable change of lung nodule volume with CT in a phantom study with high and low signal to background contrast [9783-77]

\section{Part Two}

\section{POSTER SESSION: BREAST IMAGING}

9783 2B Eigenbreasts for statistical breast phantoms [9783-82]

9783 2C Conceptual detector development and Monte Carlo simulation of a novel 3D breast computed tomography system [9783-81]

$97832 \mathrm{E} \quad$ Simulation of spiculated breast lesions [9783-84]

$97832 \mathrm{~F} \quad$ Estimation of mammary gland composition using CdTe series detector developed for photon-counting mammography [9783-85]

9783 2G Discrimination between normal breast tissue and tumor tissue using CdTe series detector developed for photon-counting mammography [9783-91]

$97832 \mathrm{H} \quad$ Biopsy system guided by positron emission tomography in real-time [9783-90]

97832 Feasibility of generating quantitative composition images in dual energy mammography: a simulation study [9783-94]

$97832 \mathrm{~J} \quad$ Comparison of contrast enhancement methods using photon counting detector in spectral mammography [9783-89]

9783 2K Grid-less imaging with antiscatter correction software in 2D mammography: the effects on image quality and MGD under a partial virtual clinical validation study [9783-92]

$97832 \mathrm{~L}$ DICOM organ dose does not accurately represent calculated dose in mammography [9783-96]

$97832 \mathrm{M} \quad$ Analysis of the scatter effect on detective quantum efficiency of digital mammography [9783-88]

$97832 \mathrm{~N} \quad$ Optimal exposure techniques for iodinated contrast enhanced breast CT [9783-83]

978320 Estimation of adipose compartment volumes in CT images of a mastectomy specimen [9783-93]

9783 2P A new paradigm of dielectric relaxation spectroscopy for non-invasive detection of breast abnormalities: a preliminary feasibility analysis [9783-95]

$97832 \mathrm{R} \quad$ Reduction of artifacts in computer simulation of breast Cooper's ligaments [9783-87] 
$97832 R \quad$ Radiation dose differences between digital mammography and digital breast tomosynthesis are dependent on breast thickness [9783-97]

POSTER SESSION: CONE BEAM CT

978325 Scatter correction in CBCT with an offset detector through a deconvolution method using data consistency [9783-105]

$97832 \mathrm{~T}$ Deblurring in iterative reconstruction of half $\mathrm{CBCT}$ for image guided brain radiosurgery [9783-107]

$97832 \mathrm{U}$ Imaging characteristics of distance-driven method in a prototype cone-beam computed tomography (CBCT) [9783-106]

$97832 \mathrm{~V}$ Lens of the eye dose calculation for neuro-interventional procedures and CBCT scans of the head [9783-101]

$97832 \mathrm{~W}$ A fast GPU-based approach to branchless distance-driven projection and back-projection in cone beam CT [9783-104]

$97832 \mathrm{X} \quad$ A system to track skin dose for neuro-interventional cone-beam computed tomography (CBCT) [9783-102]

$97832 Y \quad$ Regularization design for high-quality cone-beam CT of intracranial hemorrhage using statistical reconstruction [9783-103]

$97832 Z$ Properties of the ellipse-line-ellipse trajectory with asymmetrical variations [9783-100]

978330 Library-based scatter correction for dedicated cone beam breast CT: a feasibility study [9783-99]

978331 Ring artifacts removal via spatial sparse representation in cone beam CT [9783-98]

978332 Algorithm for x-ray beam hardening and scatter correction in low-dose cone-beam CT: phantom studies [9783-108]

\section{POSTER SESSION: CT: ARTIFACT CORRECTIONS}

978333 MADR: metal artifact detection and reduction [9783-1 10]

978334 Metal artifact reduction in CT via ray profile correction [9783-111]

978335 Line-ratio based ring artifact correction method using transfer function [9783-112]

978336 Beam hardening and motion artifacts in cardiac CT: evaluation and iterative correction method [9783-109] 
POSTER SESSION: CT: TECHNOLOGY, SYSTEM CHARACTERIZATION, DOSE, APPLICATIONS

978337 A geometric calibration method for inverse geometry computed tomography using Pmatrices [9783-113]

978338 CT dose minimization using personalized protocol optimization and aggressive bowtie [9783-114]

978339 Segmentation-free $x$-ray energy spectrum estimation for computed tomography [9783-115]

9783 3A Noise power spectrum studies of CT systems with off-centered image object and bowtie filter [9783-116]

9783 3B Prototype adaptive bow-tie filter based on spatial exposure time modulation [9783-117]

9783 3C Estimation of breast dose saving potential using a breast positioning technique for organbased tube current modulated CT [9783-1 18]

9783 3D Robust dynamic myocardial perfusion CT deconvolution using adaptive-weighted tensor total variation regularization [9783-119]

9783 3E Organ dose conversion coefficients for tube current modulated CT protocols for an adult population [9783-120]

$97833 \mathrm{~F} \quad$ A technique for multi-dimensional optimization of radiation dose, contrast dose, and image quality in CT imaging [9783-121]

9783 3G Experimental demonstration of a dynamic bowtie for region-based CT fluence optimization [9783-122]

$97833 \mathrm{H} \quad$ Hybrid deterministic and stochastic x-ray transport simulation for transmission computed tomography with advanced detector noise model [9783-123]

978331 An automated technique for estimating patient-specific regional imparted energy and dose in TCM CT exams [9783-124]

9783 3J A framework for analytical estimation of patient-specific CT dose [9783-125]

9783 3K Modulation transfer function determination using the edge technique for cone-beam micro-CT [9783-126]

9783 3L An approach for quantitative image quality analysis for CT [9783-127]

9783 3M Low dose CT perfusion using k-means clustering [9783-234] 
9783 3N Quantitative comparison using generalized relative object detectability (G-ROD) metrics of an amorphous selenium detector with high resolution microangiographic fluoroscopes (MAF) and standard flat panel detectors (FPD) [9783-128]

978330 Exploration of maximum count rate capabilities for large-area photon counting arrays based on polycrystalline silicon thin-film transistors [9783-129]

$97833 \mathrm{P}$ Indirect-detection single-photon-counting $\mathrm{x}$-ray detector for breast tomosynthesis [9783-130]

$97833 Q \quad$ SWAD: inherent photon counting performance of amorphous selenium multi-well avalanche detector (Cum Laude Poster Award) [9783-131]

$97833 R \quad$ Focal spot deblurring for high resolution direct conversion x-ray detectors [9783-133]

$978335 \quad$ Noise power spectrum measurements under nonuniform gains and their compensations [9783-134]

9783 3T A comparison of quantum limited dose and noise equivalent dose [9783-135]

$97833 \mathrm{U}$ Optimizing the Csl thickness for chest dual-shot dual-energy detectors [9783-136]

9783 3V Physical properties of a new flat panel detector with cesium-iodide technology [9783-137]

9783 3W Detective quantum efficiency: a standard test to ensure optimal detector performance and low patient exposures [9783-138]

9783 3X MTF and NPS of single-shot dual-energy sandwich detectors [9783-139]

POSTER SESSION: DUAL AND MULTI ENERGY CT

$97833 Y$ Computed tomography with single-shot dual-energy sandwich detectors [9783-140]

978332 Theoretical and Monte Carlo optimization of a stacked three-layer flat-panel x-ray imager for applications in multi-spectral diagnostic medical imaging [9783-141]

$978341 \quad$ Noise suppression for energy-resolved CT using similarity-based non-local filtration [9783-143]

978342 Dual energy $\mathbf{x}$-ray imaging and scoring of coronary calcium: physics-based digital phantom and clinical studies [9783-144]

978343 Enhanced diagnostic value for coronary CT angiography of calcified coronary arteries using dual energy and a novel high-Z contrast material: a phantom study [9783-145]

978344 Reconstruction of limited-angle dual-energy CT using mutual learning and cross-estimation (MLCE) [9783-146] 
978346 Calcium scoring with dual-energy CT in men and women: an anthropomorphic phantom study [9783-148]

978347 Dual-energy computed tomography of the head: a phantom study assessing axial dose distribution, eye lens dose, and image noise level [9783-149]

978348 Multi-energy method of digital radiography for imaging of biological objects [9783-150]

\section{POSTER SESSION: IMAGE RECONSTRUCTION}

978349 Iterative image reconstruction for multienergy computed tomography via structure tensor total variation regularization [9783-151]

9783 4A Iterative CT reconstruction using coordinate descent with ordered subsets of data [9783-152]

\section{Part Three}

9783 4B Optimization-based reconstruction for reduction of CBCT artifact in IGRT [9783-153]

9783 4C Resolution-enhancing hybrid, spectral CT reconstruction [9783-154]

9783 4D Axial 3D region of interest reconstruction using weighted cone beam BPF/DBPF algorithm cascaded with adequately oriented orthogonal butterfly filtering [9783-155]

9783 4E Joint regularization for spectro-temporal CT reconstruction [9783-156]

$97834 \mathrm{~F} \quad$ Texture-preserved penalized weighted least-squares reconstruction of low-dose CT image via image segmentation and high-order MRF modeling [9783-157]

9783 4G Regularized CT reconstruction on unstructured grid [9783-158]

$97834 \mathrm{H} \quad$ A new look at signal sparsity paradigm for low-dose computed tomography image reconstruction [9783-159]

978341 Texture-preserving Bayesian image reconstruction for low-dose CT [9783-160]

$97834 \mathrm{~J}$ Fast conjugate gradient algorithm extension for analyzer-based imaging reconstruction [9783-161]

9783 4K Efficient iterative image reconstruction algorithm for dedicated breast CT [9783-162]

9783 4L Direct reconstruction of enhanced signal in computed tomography perfusion [9783-163]

$97834 \mathrm{M} \quad$ A method for investigating system matrix properties in optimization-based CT reconstruction [9783-164]

$97834 \mathrm{~N} \quad$ A comparative study of the effects of using normalized patches for penalized likelihood tomographic reconstruction [9783-165] 
978340 High-resolution and large-volume tomography reconstruction for x-ray microscopy [9783-166]

$97834 \mathrm{P} \quad$ An adaptive method for weighted median priors in transmission tomography reconstruction [9783-167]

$97834 \mathrm{Q}$ Continuous analog of multiplicative algebraic reconstruction technique for computed tomography [9783-168]

$97834 \mathrm{R}$ Texture enhanced optimization-based image reconstruction (TxE-OBIR) from sparse projection views [9783-169]

978345 Acceleration of iterative tomographic image reconstruction by reference-based back projection [9783-170]

$97834 \mathrm{~T} \quad$ Noise reduction in computed tomography using a multiplicative continuous-time image reconstruction method [9783-171]

$97834 \mathrm{U}$ Assessment of tomographic reconstruction performance using the Mojette transform [9783-172]

POSTER SESSION: MEASUREMENTS

$97834 \mathrm{~V} \quad$ A wide-acceptance Compton spectrometer for spectral characterization of a medical xray source [9783-173]

$97834 \mathrm{~W} \quad$ X-ray spectrum estimation from transmission measurements by an exponential of a polynomial model [9783-174]

$97834 \mathrm{X}$ A dual-energy medical instrument for measurement of $\mathrm{x}$-ray source voltage and dose rate [9783-175]

\section{POSTER SESSION: NEW SYSTEMS AND TECHNOLOGIES}

$97834 Z \quad$ In vivo small animal lung speckle imaging with a benchtop in-line XPC system [9783-177]

978350 Seventh-generation CT [9783-179]

978351 A glass-sealed field emission x-ray tube based on carbon nanotube emitter for medical imaging [9783-180]

978352 Organ radiation exposure with EOS: GATE simulations versus TLD measurements [9783-181]

978354 Scattering-compensated cone beam x-ray luminescence computed tomography [9783-184]

978355 Microstructure analysis of the pulmonary acinus by a synchrotron radiation CT [9783-185] 
978356 Ultrasound waveform tomography with the second-order total-generalized-variation regularization [9783-186]

POSTER SESSION: PET, SPECT, MR, ULTRASOUND

978357 Quantitative evaluation of susceptibility effects caused by dental materials in head magnetic resonance imaging [9783-192]

978359 Effects of tissue heterogeneity on single-coil, scanning MIT imaging [9783-193]

9783 5A Quantitative evaluation of PET image using event information bootstrap [9783-195]

9783 5B Event-by-event PET image reconstruction using list-mode origin ensembles algorithm [9783-187]

9783 5C In vitro flow assessment: from PC-MRI to computational fluid dynamics including fluidstructure interaction [9783-190]

9783 5D Quantitative analysis of L-SPECT system for small animal brain imaging [9783-188]

$97835 \mathrm{E} \quad$ Characterization of various tissue mimicking materials for medical ultrasound imaging [9783-191]

POSTER SESSION: PHASE CONTRAST IMAGING

$97835 \mathrm{~F}$ Construction and evaluation of a high-energy grating-based $\mathbf{x}$-ray phase-contrast imaging setup [9783-196]

$97835 \mathrm{G}$ Feasibility of using energy-resolving detectors in differential phase-contrast imaging [9783-197]

$97835 \mathrm{H}$ Joint reconstruction of absorption and refractive properties in propagation-based x-ray phase-contrast tomography via a non-linear image reconstruction algorithm [9783-198]

$978351 \quad$ Improvement of the visibility for $x$-ray phase contrast imaging using photon counting detector [9783-199]

9783 5J Quantification of signal detection performance degradation induced by phase-retrieval in propagation-based $\mathbf{x}$-ray phase-contrast imaging [9783-200]

$97835 \mathrm{~L} \quad$ Quantitative imaging of the microbubble concentrations by using an in-line phase contrast tomosynthesis prototype: a preliminary phantom study [9783-202]

$97835 \mathrm{M}$ Evaluation of a new reconstruction algorithm for $x$-ray phase-contrast imaging [9783-203] 
POSTER SESSION: PHOTON COUNTING CT

$97835 \mathrm{~N} \quad$ Photon counting CT of the liver with dual-contrast enhancement [9783-204]

978350 Feasibility study of sparse-angular sampling and sinogram interpolation in material decomposition with a photon-counting detector [9783-207]

$97835 \mathrm{P} \quad$ Novel approaches to address spectral distortions in photon counting x-ray CT using artificial neural networks [9783-206]

$97835 \mathrm{~L} \quad$ Low-dose performance of a whole-body research photon-counting CT scanner [9783-205]

POSTER SESSION: RADIATION THERAPY

$97835 R \quad$ Estimation of the influence of radical effect in the proton beams using a combined approach with physical data and gel data [9783-209]

$97835 S$ Spot scanning proton therapy plan assessment: design and development of a dose verification application for use in routine clinical practice [9783-208]

POSTER SESSION: SCATTER AND DIFFRACTION IMAGING

$97835 \mathrm{~T} \quad$ Validation of coded aperture coherent scatter spectral imaging for normal and neoplastic breast tissues via surgical pathology [9783-211]

POSTER SESSION: TASK DRIVEN IMAGING, OBSERVERS, DETECTABILITY, PHANTOM STUDIES

9783 5V Development of a Hausdorff distance based 3D quantification technique to evaluate the CT imaging system impact on depiction of lesion morphology [9783-212]

$97835 \mathrm{X}$ Development and comparison of projection and image space 3D nodule insertion techniques [9783-217]

$97835 Y \quad$ Evaluation of a projection-domain lung nodule insertion technique in thoracic CT [9783-215]

$97835 Z$ Synthesized interstitial lung texture for use in anthropomorphic computational phantoms [9783-216]

978360 Second generation anthropomorphic physical phantom for mammography and DBT: Incorporating voxelized 3D printing and inkjet printing of iodinated lesion inserts [9783-214]

POSTER SESSION: TOMOSYNTHESIS AND DIGITAL RADIOGRAPHY

$978361 \quad$ kV x-ray dual digital tomosynthesis for image guided lung SBRT [9783-218] 
978362 Design, optimization and evaluation of a "smart" pixel sensor array for low-dose digital radiography [9783-219]

978363 Modeling acquisition geometries with improved super-resolution in digital breast tomosynthesis [9783-220]

978364 Scatter estimation and removal of anti-scatter grid-line artifacts from anthropomorphic head phantom images taken with a high resolution image detector [9783-221]

978365 Optical geometry calibration method for free-form digital tomosynthesis [9783-222]

978366 Initial clinical evaluation of stationary digital chest tomosynthesis [9783-223]

978367 Anatomical decomposition in dual energy chest digital tomosynthesis [9783-224]

978368 Optimization of exposure parameters in digital tomosynthesis considering effective dose and image quality [9783-225]

978369 Digital breast tomosynthesis reconstruction using spatially weighted non-convex regularization [9783-226]

$97836 \mathrm{~A}$ Optimal kVp in chest computed radiography using visual grading scores: a comparison between visual grading characteristics and ordinal regression analysis [9783-227]

$97836 \mathrm{~B}$ On the properties of artificial neural network filters for bone-suppressed digital radiography [9783-228]

9783 6C Effects of angular range on image quality of chest digital tomosynthesis [9783-229]

9783 6D Quantitative comparison of spatial resolution in step-and-shoot and continuous motion digital breast tomosynthesis [9783-230]

$97836 \mathrm{E} \quad \mathrm{C}$-arm technique using distance driven method for nephrolithiasis and kidney stones detection [9783-231]

9783 6F Ray tracing reconstruction investigation for C-arm tomosynthesis [9783-232]

$97836 \mathrm{G}$ Integration of kerma-area product and cumulative air kerma determination into a skin dose tracking system for fluoroscopic imaging procedures [9783-233] 


\section{Authors}

Numbers in the index correspond to the last two digits of the six-digit citation identifier (CID) article numbering system used in Proceedings of SPIE. The first four digits reflect the volume number. Base 36 numbering is employed for the last two digits and indicates the order of articles within the volume. Numbers start with 00, 01, 02, 03, 04, 05, 06, 07, 08, 09, OA, OB...0Z, followed by 10-12, 20-2Z, etc.

Aagaard-Kienitz, Beveley, OV

Abadi, Ehsan, 3F

Abou Al-Ola, Omar M., 4Q

Acciavatti, Raymond J., 03, 07,63

Agasthya, Greeshma, 3C, 3F

Ahmed, Azam S., OV

Ahn, Jeung Sun, 51

Alakhras, Maram M., 2R

Álamo, J., 2H

Albanese, K. E., 5T

Allison, Jason, $1 \mathrm{X}$

Allmendinger, Thomas, IA, IG

Allner, Sebastian, OE

Alrehily, Faisal, 2E

Anastasio, Mark A., 4Z, 5H, 5J

Andreyev, Andriy, 1N, 5B

Anton, Gisela, OP, 5F, 5M

Antonuk, Larry E., 30

Antropova, Natalia, 4K

Augustine, Kurt E., $5 S$

Aygun, N., OT, $2 Y$

Aziz, Moez Karim, 07

$\mathrm{Ba}$, Alexandre, 28

Badal, Andreu, OB, 1Z, 3B

Badano, Aldo, 14, 1Z, 32

Badea, C. T., 4C, 4E, 5P

Baek, Cheol-Ha, 2M

Bakic, Predrag R., 2O, 2Q

Barber, W., 5P

Bartels, Matthias, $1 \mathrm{H}$

Basilico, F., 57

Baturin, Pavlo, 5G

Becchetti, Marc F., 3F, $5 Z$

Bednarek, Daniel R., 1W, 2V, 2X, 3N, 3R, 64, 6G

Belian, A., 4V

Beller, Carsten J., 5C

Beltran, Chris J., $5 \mathrm{~S}$

Bemelmans, Frédéric, 2K

Benlloch, J. M., $2 \mathrm{H}$

Berggren, Karl, OA

Besson, G. M., 50

Bian, Zhaoying, 3D, 49

Bier, Elianna, 28

Binaghi, E., 57

Blevis, Ira, OX

Bochud, François, 28

Boone, John M., 3l, 4K, 61

Bosmans, Hilde, 2K

Bourne, Roger, 2R
Boyce, Sarah J., 3T

Boyd, Douglas, 61

Brady, David J., 23

Bramaje Adversalo, Xenia, 2K

Brankov, Jovan G., 4J

Brehm, Marcus, $\mathrm{OH}, 4 \mathrm{~B}$

Brennan, Patrick C., 2L, 2R

Brody, S., $4 Z$

Bruder, Herbert, 1A, 1E

Budde, Adam, 3A

Bues, Martin, $5 \mathrm{~S}$

Bysani Krishnakumar, Sumukh, IW

Calliste, Jabari, 04

Cao, Q., OK

Cao, Wanqing, 15

Carin, Lawrence, 23

Carrilero, V., $2 \mathrm{H}$

Caudevilla, Oriol, 4J

Chan, Heang-Ping, 69

Cheheltani, Rabee, IP

Chen, Arthur, 15

Chen, Baiyu, 1B, 26, 5Y

Chen, Buxin, $1 \mathrm{~K}$

Chen, Elvis C. S., 5E

Chen, Guang-Hong, OM, ON, OU, OV, OW, 10, 1D, $3 \mathrm{~A}$

Chen, Jun, 62

Chen, Ting, $0 \mathrm{C}$

Chen, Wei R., 5L

Chen, Yang, 1D

Chen, Ying, 6E, 6F

Chen, Yujia, $5 \mathrm{H}$

Chen, Yun, 4G

Cheng, Chang-Chieh, 4O, 4S

Ching, Yu-Tai, 4O, 4S

Cho, Min Kook, $3 \mathrm{~J}$

Cho, Seungryong, 1U, 2 S

Choi, Jang-Hwan, OL

Choi, Mina, 12

Choi, Seungyeon, 21, 67, 68, 6C

Choi, Sunghoon, 2I, 2U, 67, 68, 6C

Chou, Cheng-Ying, 5J

Christensen, Soren, OY

Chtcheprov, Pavel, 65

Chung, Yong Hyun, 5A

Clark, D. P., 4C, 4E, 5P

Clark, Matthew, $\mathrm{OB}$

Clavel, A. H., 52

Cochran, Joe, 3L 
Coradi, Thomas, 11

Cormode, David, IP

Correcher, C., $2 \mathrm{H}$

Cruz-Bastida, Juan P., 3A

Cunningham, Ian A., 18, 3W, $3 Z$

Daerr, Heiner, $1 \mathrm{H}, 5 \mathrm{~N}$

Daly, Megan, 61

Dance, David R., 06, 08, 2E

Dang, H., OT, $2 Y$

Danielsson, Mats, OA

Darbon, A., 52

Das, Mini, $0 Q$

Davis, Brian J., 02

De Carlo, Francesco, 5H

De Man, Bruno, 38, $3 \mathrm{G}$

Der Sarkissian, Henri, $4 \mathrm{U}$

Dhurjaty, Sreeram, 2P

Diao, Andrew, 28, 60

Dietrich, O., 10

Diffey, Jennifer, 2R

Divel, Sarah E., OY

Doki, T., 5 I

Dunkerley, David A. P., 1Y, 37

Durst, Roger, 3P

Elangovan, Premkumar, 06, 08, 2E

El-Mohri, Youcef, 30

Eriksson, Markus, 2T

Escartin, Terenz R., 18, 3W

Espinoza, Camilo, $1 \mathrm{X}$

Espy, Michelle A., 4V

Evans, Joshua D., $\mathrm{OZ}$

Fahrig, Rebecca, OL

Fang, Yuan, 14

Fatyga, Mirek, 5S

Favazza, Christopher, 26

Fayad, Zahi A., IP

Feldkamp, J. R., 59

Feng, Qianjin, 3D, 49

Ferretti, R., 57

Fessler, Jeffrey A., 69

Fiebich, Martin, $3 \mathrm{~V}$

Fieselmann, Andreas, 0G

Fingerle, Alexander A., $5 \mathrm{~N}$

Fitzgerald, Paul, $3 G$

Fleischmann, Christof, Ol

Fletcher, Joel G., 1B, 5Y

Flohr, Thomas, 1A, 1E, 1G, 10

Foos, David, OT, 2Y, 66

Fredenberg, Erik, OA

Fredette, Nathaniel R., $O Q$

Freeman, Matthew S., $1 X$

Fu, Wanyi, 3C, 3E

Funk, Tobias, $1 Y$

Gang, Grace, 0X, 24

Gao, Bo, 04

Gao, Kai, 0C

Gao, Peng, 32, 3K, 54

Garrett, John, OW

Garson III, A. B., 4 Z

Gaver, Tobias, 13
Gavrielides, Marios A., 29, 46

Ge, Yongshuai, OM, ON

Gehm, Michael E., 28, 60

Gehring, A., 4V

Ghammraoui, Bahaa, OB, 1 Z

Ghani, Muhammad U., 5L, 6D

Ghielmi, M., 57

Gilat-Schmidt, Taly, 11, 1S

Gilkeson, Robert C., 42

Glick, Stephen J., 25, 27, 2N, 60

Goett III, John Jerome, 1X

Gold, Garry, OL

Goldan, Amir H., 17, 3Q

Gomez, J., 2H

Gomez-Cardona, Daniel, 3A

Gong, Changfei, 3D, 49

Gong, Qi, 29

Goodsitt, Mitchell M., 3C

Graff, Christian G., 09

Greenberg, Joel A., 21, 23, 5T

Guédon, Jeanpierre, 4U

Gunsten, S., $4 Z$

Guo, Zijia, $2 Z$

Gursoy, Doga, 5H

Gutjahr, R., 1G, 10

$\mathrm{Ha}$, Sungsoo, 33, 34

Haas, Benjamin, 11

Hadjipanteli, Andria, 06

Hahn, Andreas, $\mathrm{OH}, \mathrm{OS}$

Hahn, Andreas, 3V

Hahn, Juliane, IA

Hahn, Katharina, 1G, 1I, 10, 4A, 5Q

Haines, T., 4V

Halaweish, Ahmed F., 11, 5Q

Hamaguchi, Takashi, 47

Han, Hao, 4F, 4I

Haneda, K., 5R

Hanson, Kenneth, OC

Harman, Allison, 05

Harms, Joe, 41

Harrigan, Robert L., $1 \mathrm{~L}$

Hartman, Allison E., 65, 66

Hashemi, SayedMasoud, 2T

Hassan, Mehadi, 23

Hatt, Charles R., 37

Hauke, Christian, OP, 5F, 5M

$\mathrm{He}$, Ji, 49

Heath, Michael, 66

Hellingman, D., 2H

Henning, Andre, 11, 5Q

Henzler, Thomas, 3M

Hernandez, Andrew, 61

Hertz, Hans M., IV

Heuveline, Vincent, 5C

Hoeschen, Christoph, 2C

Hofmann, Christian, 13

Hogan, Gary, $1 X$

Hollander, Brian, $1 \mathrm{X}$

Holmes III, David, 1B

Holmgren, Andrew, 23

xviii

Proc. of SPIE Vol. $9783978301-18$ 
Horiba, A., 5

Horn, Florian, OP, 5F, 5M

Howansky, Adrian, 17

Hsieh, Jiang, 1D, 3A

Hsieh, Scott S., OJ, OL, 1C

$\mathrm{Hu}$, Yifan, 4l

$\mathrm{Hu}$, Zhiqiang, $1 \mathrm{~N}$

Huang, Jing, 3D, 49

Huang, Lianjie, OC, 56

Hunter, J., 4V

Hwang, Dosik, 35

Hwu, Yeukuang, 40

Ichikawa, Katsuhiro, 47

Ihori, Akiko, 2F, 2G

Ikejimba, Lynda, 25, 27

Imran, Abdullah-Al-Zubaer, 20

Intrator, Miranda, $0 \mathrm{C}$

Ionita, Ciprian N., 1W, 3N, 3R

Itoh, H., 55

Jafari, Houman, 04

Jain, Amit, 1W, 2V, 3N, 64

Jaiswal, Sunil Prasad, 33

James, M., 4V

Jang, Dong-Hyuk, 68

Jeon, Hosang, 2M, 3J, 3U, 3Y, 6B

Jeon, Pil-Hyun, 68

Jeong, Jaeik, 51

Jerebko, Anna K., OG

Jiang, Hao, 3P

Jin, Yannan, 38

Jo, Byungdu, 2J, 50

Job, Isaias D., 3T

Jorgensen, S., 11

Jost, G., $1 G$

Jung, Ji Eun, 4P

Kachelriess, Marc, $\mathrm{OH}, \mathrm{OI}, \mathrm{OS}, 1 \mathrm{~A}, 3 \mathrm{M}$

Kaercher, Joerg, 3P

Kaganovsky, Yan, 23

Kalra, Mannudeep K., 38

Kang, Dong-Joong, 6B

Kang, Joo Hyun, 5A

Kao, Chien-Min, $1 \mathrm{~K}$

Kapadia, Anuj J., 21, 5T

Kappler, Steffen, 1F, 1G, 1I, 10, 1R, 5Q

Karellas, Andrew, 30

Karim, Karim S., 18, 3N, $3 Z$

Kato, Misa, 2F, 2G

Kawamoto, Satomi, $0 \mathrm{X}$

Kawashima, Hiroki, 47

Kawata, Y., 55

Kazerooni, Ella A., 3C

Ketcha, M., 2B

Kim, Changhwan, 2S

Kim, Daecheon, 3Y, 6B

Kim, Dohyeon, 2J, 50

Kim, Dong Sik, 35

Kim, Dong Woon, 2M, 3U, 3X, 3Y

Kim, Eun, 3S

Kim, Hee-Joung, 2I, 2J, 2U, 5O, 67, 68, 6C

Kim, Ho Kyung, 2M, 3J, 3K, 3U, 3X, 3Y, 6B
Kim, Hyemi, 2J, 50

Kim, Jin Sung, 6B

Kim, Jin Woo, 3J

Kim, Junwoo, 3U, 3X

Kim, Kyeong Min, 5A

Kim, Myeongsoo, 6A

Kim, Namho, 61

Kim, Seung Ho, 3Y

Kim, Seung Hoon, 51

Kim, Ye-seul, 21, 2U, 67, 68, 6C

Kim, Younguk, 35

Kimura, K., 5l

Klasky, M., 4V

Klotz, Ernst, 3M

Knaup, Michael, 01

Kobayashi, Masanao, 47

Kodera, Yoshie, 2F, 2G

Kofler, James, 26

Kojima, Takeshi, 4Q, 4T

Koliatsos, V. E., OT, 2 Y

Koniczek, Martin, 30

Koo, Chi Wan, 5 Y

Koshida, Kichiro, 47

Kratzke, Jonas, 5C

Krauss, B., $1 \mathrm{G}$

Kuntz, Jan, Ol

Kuperavage, Adam, $2 Q$

Kuzo, Ronald S., 5 Y

Kwak, Junghwan, 51

Kwak, Shin Hye, 5A

Kwiatkowski, Kris, $1 \mathrm{X}$

Kwon, Young Joon, 03, 07

Lachner, Sebastian, OP, 5F, 5M

Laganis, Philip E., 04

Lakshmanan, Manu N., 21, 5 T

Lambert, Jack W., 43

Landman, Bennett A., $1 \mathrm{~L}$

Lane, J., 11

Lansberg, Maarten G., OY

Larsson, Daniel H., IV

Larsson, Jakob C., IV

Lau, Kristen C., 07

Lauritsch, Guenter, $2 Z$

Lee, Dong-Hoon, 2I, 2U, 67, 68, 6C

Lee, Haenghwa, 2I, 2U, 67, 68, 6C

Lee, Hoyeon, $2 S$

Lee, Okkyun, 1R, 36

Lee, Soo-Jin, 4N, 4P

Lee, Young, 2T

Lee, Yueh Z., 04, 05, 65, 66

Lell, Michael, OS

Leng, Shuai, 12, 1B, 1F, 11, 26, 5Q, 5Y

Létang, J. M., 52

Levéille, Sébastien, 17

Levenston, Marc, OL

Levin, David L., 1I, 5Y

Levinson, Reuven, OX

$\mathrm{Li}$, Bin, $4 \mathrm{~L}$

Li, Guang, 31

Li, Ke, OM, ON, OU, 10, 1D, 3A 
Li, Lihong, 4I

Li, Ping-Hui, 4S

Li, Qin, 29, 46

Li, Yinsheng, OU, OV, OW, ID

Li, Yuhua, 5L

Li, Zhongyuan, 31

Li, Zhoubo, 12, 1F, 11, 5Q

Liang, Albert K., 30

Liang, Zhengrong, 3D, 49, 4F, 4H, 4I

Liao, Qimei, 32, 3K, 54

Lin, Youzuo, OC, 56

Lipkin, Michael E., 6 E

Liu, Hong, 5L, 6D

Liv, Langechuan, 15

Liv, Songtao, 46

Liu, Wenlei, 32, 3K, 54

Liv, Xinghui, 62

Liv, Yan, 4H, $4 \mathrm{I}$

Lo, Joseph Y., 25, 27, 28, 2B, 60

Lomako, Andrey, 16

Looney, Padraig T., 06

Lopez Maurino, Sebastian, $3 z$

Lopez, Julian, $1 \mathrm{X}$

Lu, HongBing, 32, 3K, 54

Lu, Jianping, 04, 05, 65, 66

Lu, Lijun, 3D, 49

Lu, Minghui, 15

Lu, Yao, 4G

Ludwig, Veronika, OP, 5F, 5M

Lundström, Ulf, IV

Luo, Shouhua, 31

Lyu, Qingwen, 4L

Ma, Chi, $5 Y$

Ma, Jianhua, 3D, 49, 4l, 4L

Ma, Xiangyuan, 4G

Macchi, A., 57

Mackenzie, Alistair, 06

Maeda, K., 55

Maidment, Andrew D. A., 03, 07, 2O, 2Q, 63

Maier, Andreas, OL, $2 \mathrm{Z}$

Makeev, Andrey, 27, 2N

Makhota, S., 4X

Malalla, Nuhad A. Y., 6E, 6F

Mariam, Fesseha, $1 \mathrm{X}$

Marshall, Nicholas, 2K

Martinez, Michael, 1X

Mathews, Aswin, OX

Matsubara, Kosuke, 47

Mawn, Louise A., $1 \mathrm{~L}$

McCall, S. J., 5T

McCollough, Cynthia H., 12, 1B, 1F, 11, 26, 5Q, 5Y

McEntee, Mark F., 2 L

Mechlem, Korbinian, $\mathrm{OE}$

Medeiros, Henry, 2W

Medina, Jason, IX

Medina, Patrick, IX

Mei, Kai, OE

Mello-Thoms, Claudia, 2R

Mendez, J., 4V

Merrill, Frank, 1X
Mertelmeier, Thomas, OG

Michel, Thilo, OP, 5F, 5M

Miller, James J., 16

Miller, M. I., 2B

Minami, K., 55

Mistretta, Charles, 02

Moir, D., 4V

Moliner, L., 2H

Mooney, Doug, 3L

Moore, William, 4F, 4H, 4I

Morley, Deborah, IX

Morris, Chris, IX

Morris, Robert E., 21, 5T

Mueller, Klaus, 33, 34

Muenzel, Daniela, $5 \mathrm{~N}$

Müller, Bernhard H., 2C

Muller, Kerstin, OL

Mundy, Daniel W., 5S

Munro, Peter, 4B

Murray, Matthew, $1 \mathrm{X}$

Musinsky, Stephanie, 60

Myers, Kyle, 29, 46

Nakajima, Ai, 2F, $2 \mathrm{G}$

Nakano, Y., 55

Nam, Jiho, 3J

Nam, Wooseok, 2T

Nano, Tomi F., 18, 3W

Naydenov, S. V., 48, 4X

Nedrow, Paul, $1 \mathrm{X}$

Neumann, Bernd, 2C

Niemann, David B., OV

Niki, N., 55

Niu, Kai, OU, OV

Niu, Tianye, 39, 4R

Noël, Peter B., OE, 5N

Noh, Eunkyong, 51

Noo, Frédéric, 2Z, 4A

Nordström, Håkan, 2T

Normand, Nicolas, $4 \mathrm{U}$

Novario, R., 57

Nye, Katelyn, 42

Oberstar, Erick L., 02

Odinaka, Ikenna, 23

O'Donnell, Thomas, IP

Oh, Daejoong, 35

Ohmatsu, H., 55

Okada, Masahiro, 2F, $2 \mathrm{G}$

Okamoto, Chizuru, 2F, 2G

Olson, Kyle, 04

Opolonin, O. D., 48, 4X

Ordovas, Karen G., 43

Orero, A., 2H

O'Sullivan, Joseph A., 23

Ou, Hai, 62

Paik, Sanghyun, 51

Pan, Xiaochuan, 1K, 4B, 4K, 4M, 4W

Park, Chan-Soo, OQ

Park, Eunpyeong, 6B

Park, Hunkuk, 51

Park, Jiwoong, 2M 
Park, Junbeom, 6B

Park, Miran, 2S

Park, Subok, 2B

Park, Su-Jin, 2J, 50

Parry, Mark D., 5S

Partain, Larry, 61

Paysan, Pascal, $\mathrm{OH}, 4 \mathrm{~B}$

Pearce, Caleb, 05

Pelc, Norbert J., OY, 1C, 1J, 1Q

Pelizzari, Charles, 4B

Pelzer, Georg, OP, 5F, 5M

Penchev, Petar, 3V

Peng, Luke, 15

Peris, J. L., 2H

Perkhounkov, Boris, 4W

Perkins, Amy E., $1 \mathrm{~K}$

Peters, Inge M., 16

Peters, Terry M., 5E

Petrick, Nicholas, 29, 46

Petrillo, Michael J., 3T

Petrongolo, Michael, 41

Pfeiffer, Franz, OE, 5N

Pickering, Mark R., 5D

Pietsch, H., $1 G$

Pinto, R. Ferrari, 2E

Pisana, Francesco, 3M

Pochet, T., 4X

Podgorsak, Alexander R., IW

Poepping, Tamie, 5E

Pokrajac, David D., 2O, 2Q

Politte, David G., 23

Polster, Christoph, 1G, 10, 1R

Popescu, Lucretiu M., 3H

Porras-Chaverri, Mariela A., $\mathrm{OZ}$

Potuzko, Marci, 05

Pourmorteza, A., 19

Preminger, Glenn M., 6E

Proksa, Roland, 1H, 5N

Pu, Huangsheng, 54

Qian, Wei, 2P

Qin, Bin, ON

Qin, Jun, 6E

Qiu, Yuchen, 2P

Quirk, S., 59

Rahimi, Amir, 3L

Rahman, Tasneem, 5D

Rajbhandary, Paurakh L., IQ

Rana, R., 3R, 64

Rana, Vijay, 2V

Rashidnasab, Alaleh, 2E

Ratnanather, J. T., 2B

Recur, Benoit, $4 \mathrm{U}$

Regensburger, Joe, 3L

Reiser, Ingrid S., 4K

Ren, Liqiang, 6D

Ren, Xue, 4N

Ren, $Y i, 4 R$

Rengier, Fabian, 5C

Rickard, Mary, $2 \mathrm{R}$

Rieger, Jens, OP, 5F, 5M
Ritman, Erik L., 11, 5Q

Ritschl, Ludwig, OG

Robins, Marthony, 5V, $5 \mathrm{X}$

Robinson, Vance, 3G

Roessl, Ewald, $1 \mathrm{H}$

Rohkohl, C., $1 \mathrm{E}$

Rong, Junyan, 32, 3K, 54

Rose, Sean D., 1K, 4M

Royalty, Kevin, OU

Rudin, Stephen, 1W, 2V, 2X, 3N, 3R, 64, 6G

Rui, Xue, 3G

Rummeny, Ernst J., 5N

Ruschin, Mark, 2T

Russ, M., 3N, 3R

Ryu, Jehwang, 51

Ryzhikov, V. D., 48, 4X

Sahbaee, Pooyan, 3E, 3F, 5V, 5X

Sahgal, Arjun, $2 T$

Sahiner, Berkman, 29, 46

Sakai, H., 55

Salvagnini, Elena, $2 \mathrm{~K}$

Samei, Ehsan, 21, 25, 28, 3C, 3E, 3F, 3I, 5V, 5X, 5Z, 60

Sanchez, Adrian, 4K

Sanders, Jeremiah W., 3F, 31

Sano, S., 51

Sato, T., 51

Saunders, Alexander, $1 \mathrm{X}$

Sauppe, Sebastian, $\mathrm{OH}, \mathrm{OS}$

Schafer, Sebastian, OU

Schevermann, James R., 17

Schlifske, Daniel, 2W

Schmidt, Bernhard, 1G, 3M

Schoeck, Friederike, 1G, 10, 1P

Scholz, Rosemarie, OG

Schönberg, Stefan, 3M

Schöndube, H., 4A

Schurman, Tamsen, $1 \mathrm{X}$

Schuster, Max, OP, 5F, 5M

Scott, C., 3N

Sedillo, R., 4V

Sedlmair, M., $1 G$

Segars, William Paul, OY, 2B, 3C, 3E, 3F, 3I, $5 Z$

Seghers, Dieter, $\mathrm{OH}, 4 \mathrm{~B}$

Seifert, Maria, $O P, 5 F, 5 M$

Seo, Chang-Woo, $2 \mathrm{U}$

Setlur Nagesh, S. V., 1W, 3N, 3R

Shan, Jing, 05, 65, 66

Shankar, Alok, 3N, 64, 6G

Shen, Zeyang, 36

Shi, Linxi, 30

Shin, Choul Woo, 35

Shin, Junseob, 0C

Shirai, T., 5 I

Shunhavanich, Picha, $1 \mathrm{~J}$

Shurter, R., 4V

Sidky, Emil Y., 1K, 4B, 4K, 4M, 4W

Siewerdsen, Jeffrey H., OK, OR, OT, OX, 19, 24, $2 Y$

Sikaria, Dhiraj, 60

Sisneros, Thomas, $1 \mathrm{X}$ 
Sisniega, A., OK, OT, $2 Y$

Slagowski, Jordan M., 37

Smit, Chiel, 16

Smith, Alex K., $1 \mathrm{~L}$

Smith, C. F., 48, 4X

Smith, Seth A., $1 \mathrm{~L}$

Smith, Walt, 3G

Solomon, Justin B., 28, 5V, 5X, 5Z, 60

Song, Bongyong, $2 \mathrm{~T}$

Song, Hankyeol, 5A

Song, William, 2T

Song, Xiyun, $1 \mathrm{M}, 1 \mathrm{~N}$

Soriano, Carmen, $5 \mathrm{H}$

Speidel, Michael A., 02, 1Y, 37

Spronk, Derrek, 04

Star-Lack, Josh, 0J, 11

Stavro, Jann, 3Q

Stayman, J. Webster, OK, OR, OT, OX, 19, 24, 2Y

Stearns, J., $4 \mathrm{~V}$

Stec, Jessika, 4W

Steinbrener, Jan, OG

Stierstorfer, Karl, 1A, 1E, 4A

Stoker, Joshua B., 5 S

Strocchi, S., 57

Strother, Charles, 02, OU, OV

Sturgeon, Gregory M., 2B, 3C, 60

Suleiman, Moayyad E., 2 L

Sun, Pengfei, 6E

Sun, Yi, 31

Sun, Yuxin, 43

Taguchi, Katsuyuki, 1R, 36

Tahtali, Murat, 5D

Tainter, Amy, IX

Takahashi, Edwin A., 5 Y

Takata, Tadanori, 47

Tan, Maxine, $2 \mathrm{P}$

Tanabe, K., 5

Tang, Shaojie, 4D

Tang, Xiangyang, 4D, 4R

Tanioka, K., 17

Tao, Kun, 38

Tateishi, Kiyoko, $4 Q$

Tattersall, P., $2 \mathrm{H}$

Thevenard-Berger, P., 52

Thouvenot, Audrey, 5E

Tian, Xiaoyu, 3C, 3E, 3I

Tilley, Steven, OR

Touch, M., 5P

Tousignant, Olivier, 17

Trouw, Frans, $1 \mathrm{X}$

Tung, Chi-Hua, IK

Tupa, Dale, $1 \mathrm{X}$

Tward, Daniel J., 2B

Tybo, Josh, $1 \mathrm{X}$

Umetani, K., 55

Vågberg, William, $1 \mathrm{~V}$

Van Ongeval, Chantal, 2K

Van Peteghem, Nelis, 2K

Van Syoc, K., 4V

Vasireddi, S., $4 Z$
Vedantham, Srinivasan, 30

Vent, Trevor L., 03

Verdun, F. R., 52

Viers, Lyndsay D., $5 Y$

Vijayan, Sarath, 2V, 2X, 6G

Villanueva, Flordeliza, $\mathrm{ON}$

Vincent Sheldon, Stephanie A., 5 Y

Vogt, Carmen, $1 \mathrm{~V}$

Voigt, Johannes M, OG

Volegov, P., 4V

Volkov, V. G., 48, 4X

Vrieze, Thomas, 26

Walsh, Timothy J., $5 \mathrm{~S}$

Wandner, Johannes, OP, 5F, 5M

Wang, Adam S., 0J, 11

Wang, Hui, 38

Wang, Jing, 4L

Wang, Jiong, 05

Wang, Kai, 62

Wang, Kun, $5 \mathrm{H}$

Wang, Tonghe, 41

Wang, Xiaohui, OT, 2Y, 66

Weber, Thomas, 5F

Wei, Xinzhou, 4F

Weis, Christian, 5C

Wells, Kevin, 06, 08, 2E

Wen, Di, 42

Werner, René, 13

Whiting, Bruce R., ON, OZ

Wi, Sunhee, $1 \mathrm{U}$

Wilde, Carl, 1X

Wileyto, E. Paul, 63

Williamson, Jeffrey $\mathrm{F} ., \mathrm{OZ}$

Wilson, David L., 42

Wintermark, Max, OY

Wolf, Andreas, OP, 5F, 5M

Wong, Molly D., 5L, 6D

Woo, Sang-Keun, 5A

Wu, Di, 5L, 6D

Wu, Gongting, 04, 05, 66

Wu, Mingye, 38, 3G

Wu, Xizeng, 6D

Xia, Dan, 1K, 4B

Xie, Huiqiao, 4R

Xing, Yuxiang, OD, 44

Xiong, Zhenyu, 2V, 2X

$X U$, J., OT, $2 Y$

$X u$, Yuesheng, 4G

Yamaguchi, Yusaku, 4Q, 4T

Yamakawa, Tsutomu, 2F, 2G

Yamamoto, Shuichiro, 2F, 2G

Yang, Kai, 5L, 6D

Yang, Pengfei, OV

Yang, Sook, 6A

Yang, $Y i, 4 R$

Yao, Yangyang, 38

Ye, Jinghan, $1 \mathrm{~K}, 1 \mathrm{M}, 1 \mathrm{~N}$

Yeh, Benjamin M., 43

Yeo, Seung Jun, 51

Yin, Zhye, 38, 3G 
Yorkston, J., OK

Yoshimuta, T., $5 \mathrm{I}$

Yoshinaga, Tetsuya, 4Q, 4T

Youn, Hanbean, 2M, 3J, 3U, 3X, 3Y, 6B

Young, Kenneth C., 06, 08, 2E

Yu, Lifeng, 12, 1B, 26, 5Y

Yu, Zhicong, 1F, 1I, 5Q

Yun, Seungman, 2M, 3J

Zbijewski, Wojciech, OK, OR, OT, OX, $2 Y$

Zeng, Dong, 3D, 49

Zeng, Rongping, 29, 46

Zhang, HaO, 4F, 4H, 4I

Zhang, Hua, 3D, 49

Zhang, Huayu, OD, 44

Zhang, Qiude, 39

Zhang, Ran, OM, ON

Zhang, Xinyu, 3D

Zhang, Yakun, 3E, 3F

Zhang, Zheng, 1K, 4B

Zhao, Qihua, 30

Zhao, Wei, 17, 3Q

Zhao, Wei, 39

Zheng, Bin, 2P, 5L, 6D

Zheng, Jiabei, 69

Zheng, Xiaoming, 6A

Zhou, Bo, 42

Zhou, Kungang, 3T

Zhou, Otto, 04, 05, 65, 66

Zhu, Lei, 30, 41

Zhu, Yang-Ming, $1 \mathrm{~N}$

Ziegle, Jens, 2C

Zimmerman, Kevin C., is 
Proc. of SPIE Vol. $9783978301-24$

Downloaded From: https://www.spiedigitallibrary.org/conference-proceedings-of-spie on 26 Apr 2023 Terms of Use: https://www.spiedigitallibrary.org/terms-of-use 


\section{Conference Committee}

Symposium Chairs

Steven C. Horii, The University of Pennsylvania Health System

(United States)

Berkman Sahiner, U.S. Food and Drug Administration (United States)

Conference Chairs

Despina Kontos, The University of Pennsylvania Health System

(United States)

Thomas G. Flohr, Siemens Healthcare GmbH (Germany)

Conference Co-chair

Joseph Y. Lo, Duke University Medical Center (United States)

Conference Program Committee

Andreu Badal, U.S. Food and Drug Administration (United States)

Kirsten Boedeker, Toshiba Medical Research Institute USA

(United States)

Hilde Bosmans, Katholieke Universiteit Leuven (Belgium)

Guang-Hong Chen, University of Wisconsin-Madison (United States)

Mini Das, University of Houston (United States)

Mats E. Danielsson, KTH Royal Institute of Technology (Sweden)

Maria Drangova, Robarts Research Institute (Canada)

Rebecca Fahrig, Siemens Healthcare GmbH (Germany) and Stanford University School of Medicine (United States)

Taly Gilat-Schmidt, Marquette University (United States)

Stephen J. Glick, U.S. Food and Drug Administration (United States)

and University of Massachusetts Medical School (United States)

Michael Grass, Philips Research (Germany)

Christoph Hoeschen, Otto von Guericke Universität Magdeburg

(Germany)

Marc Kachelriess, Deutsches Krebsforschungszentrum (Germany)

Karim S. Karim, University of Waterloo (Canada)

Hee-Joung Kim, Yonsei University (Korea, Republic of)

Jinyi Qi, University of California, Davis (United States)

Magdalena Rafecas, Universität zu Lübeck (Germany)

Ingrid S. Reiser, The University of Chicago (United States)

John A. Rowlands, Thunder Bay Regional Research Institute (Canada)

John M. Sabol, GE Healthcare (United States) 
Joseph W. Stayman, Johns Hopkins University (United States)

Anders Tingberg, Lund University (Sweden)

John Yorkston, Carestream Health, Inc. (United States)

Wei Zhao, Stony Brook Medicine (United States)

\section{Session Chairs}

1 Tomosynthesis and Digital Subtraction Angiography

Joseph Y. Lo, Duke University Medical Center (United States)

John M. Sabol, GE Healthcare (United States)

2 Breast Imaging

Andreu Badal, U.S. Food and Drug Administration (United States)

Wei Zhao, Stony Brook Medicine (United States)

3 Keynote and Dual and Multi Energy CT

Despina Kontos, The University of Pennsylvania Health System

(United States)

Thomas G. Flohr, Siemens Healthcare GmbH (Germany)

4 Cone Beam CT I: New Technologies, Corrections

John Yorkston, Carestream Health, Inc. (United States)

Hilde Bosmans, Katholieke Universiteit Leuven (Belgium)

5 Phase Contrast Imaging

Mini Das, University of Houston (United States)

Guang-Hong Chen, University of Wisconsin-Madison (United States)

6 Cone Beam CT II: System Optimization, Image Reconstruction

Marc Kachelriess, Deutsches Krebsforschungszentrum (Germany)

Maria Drangova, Robarts Research Institute (Canada)

7 CT I: Technology, System Characterization, Applications

Christoph Hoeschen, Otto von Guericke Universität Magdeburg (Germany)

Kirsten Boedeker, Toshiba Medical Research Institute USA, Inc.

(United States)

8 Detectors

Karim S. Karim, University of Waterloo (Canada)

Rebecca Fahrig, Siemens Healthcare GmbH (Germany) and Stanford University School of Medicine (United States)

9 CT II: Image Reconstruction, Artifact Reduction

Joseph W. Stayman, Johns Hopkins University (United States)

Michael Grass, Philips Research (Germany) 
10 Photon Counting CT I: Instrumentation

Stephen J. Glick, U.S. Food and Drug Administration (United States)

Norbert J. Pelc, Stanford University (United States)

11 PET and MR

Jinyi Qi, University of California, Davis (United States)

Christoph Hoeschen, Otto von Guericke Universität Magdeburg

(Germany)

12 Photon Counting CT II: Spectral Imaging

Taly Gilat-Schmidt, Marquette University (United States)

Norbert J. Pelc, Stanford University (United States)

13 New Systems and Technologies

Ingrid S. Reiser, The University of Chicago (United States)

Wei Zhao, Stony Brook Medicine (United States)

14 Scatter and Diffraction Imaging

Mini Das, University of Houston (United States)

Guang-Hong Chen, University of Wisconsin-Madison (United States)

15 Task Driven Imaging, Observers, Detectability, Phantom Studies

Hee-Joung Kim, Yonsei University (Korea, Republic of)

Anders Tingberg, Lund University (Sweden) 
Proc. of SPIE Vol. $9783978301-28$

Downloaded From: https://www.spiedigitallibrary.org/conference-proceedings-of-spie on 26 Apr 2023 Terms of Use: https://www.spiedigitallibrary.org/terms-of-use 


\section{Medical Imaging Award Recipients}

\section{Robert F. Wagner Best Student Paper Award}

Robert F. Wagner was an active scientist in the SPIE Medical Imaging meeting, starting with the first meeting in 1972 and continuing throughout his career. He ensured that the $\mathrm{BRH}$, and subsequently the $\mathrm{CDRH}$, was a sponsor for the early and subsequent Medical Imaging meetings, helping to launch and ensure the historical success of the meeting. The Robert F. Wagner All-Conference Best Student Paper Award (established 2014) is acknowledgment of his many important contributions to the Medical Imaging meeting and his many important advances to the field of medical imaging.

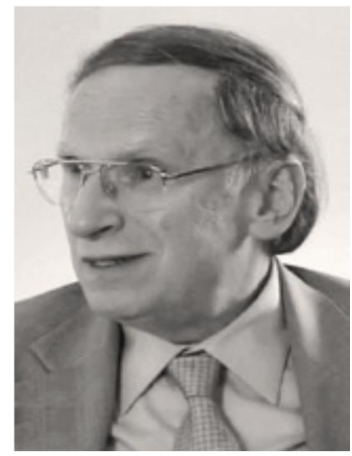

This award is cosponsored by:

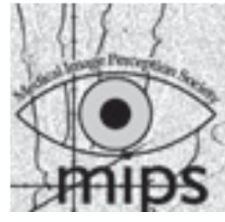

The Medical Image Perception Society

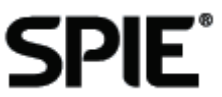

\section{Recipients:}

First Place: MIND Demons for MR-to-CT deformable image registration in imageguided spine surgery (9786-16)

S. Reaungamornrat, T. De Silva, A. Uneri, Johns Hopkins Univ. (United States), J.-P. Wolinsky, Johns Hopkins Hospital (United States), A. J. Khanna, Johns Hopkins Health Care \& Surgery Ctr. (United States), G. Kleinszig, S. Vogt, Siemens Healthcare (Germany), J. L. Prince, J. H. Siewerdsen, Johns Hopkins Univ. (United States)

Second Place: Design, fabrication, and implementation of voxel-based 3D printed textured phantoms for task-based image quality assessment in CT (9783-76)

Justin Solomon, Duke Univ. School of Medicine (United States), Alexandre Ba, Institut Univ. de Radiophysique Appliquée (Switzerland), Andrew Diao, Duke Univ. (United States), Joseph Lo, Elianna Bier, Duke Univ. School of Medicine (United States), François Bochud, Institut Univ. de Radiophysique Appliquée (Switzerland), Michael Gehm, Duke Univ. (United States), Ehsan Samei, Duke Univ. School of Medicine (United States)

\section{Conference 9786 Awards}

\section{Young Scientist Awards sponsored by Siemens}

First Place: MIND Demons for MR-to-CT deformable image registration in imageguided spine surgery (9786-16)

S. Reaungamornrat, T. De Silva, A. Uneri, Johns Hopkins Univ. (United States), J.-P. Wolinsky, Johns Hopkins Hospital (United States), A. J. Khanna, Johns Hopkins Health 
Care \& Surgery Ctr. (United States), G. Kleinszig, S. Vogt, Siemens Healthcare

(Germany), J. L. Prince, J. H. Siewerdsen, Johns Hopkins Univ. (United States)

Runner Up: Image-guided preoperative prediction of pyramidal tract side effect in deep brain stimulation (9786-29)

C. Baumgarten, Y. Zhao, INSERM (France), LTSI, Univ. de Rennes 1 (France); P. Sauleau, C. Malrain, Ctr. Hospitalier Univ. de Rennes (France); P. Jannin, INSERM (France), LTSI, Univ. de Rennes 1 (France); C. Haegelen, INSERM (France), LTSI (France), Ctr. Hospitalier de Rennes (France)

Runner Up: Increasing safety of a robotic system for inner ear surgery using probabilistic error modeling near vital anatomy (9786-51)

Neal P. Dillon, Michael A. Siebold, Jason E. Mitchell, Vanderbilt Univ. (United States), Gregoire S. Blachon, Ramya Balachandran, Vanderbilt Univ. Medical Ctr. (United States), J. Michael Fitzpatrick, Vanderbilt Univ. (United States), Robert J. Webster III, Vanderbilt Univ. Medical Ctr. (United States)

\section{Cum Laude Poster Award}

First Place: Patch-based label fusion for automatic multi-atlas-based prostate segmentation in MR images (9786-72)

Xiaofeng Yang, Ashesh B. Jani, Peter J. Rossi, Hui Mao, Walter J. Curran, Tian Liu, Winship Cancer Institute, Emory Univ. (United States) 